Published in final edited form as:

Int Rev Neurobiol. 2009 ; 84: 81-103. doi:10.1016/S0074-7742(09)00405-X.

\title{
CONTRIBUTIONS OF NEUROPSYCHOLOGY AND
}

\section{NEUROIMAGING TO UNDERSTANDING CLINICAL SUBTYPES OF MILD COGNITIVE IMPAIRMENT}

\author{
Amy J. Jak ${ }^{*}, \dagger$ Katherine J. Bangen ${ }^{*}$, Christina E. Wierenga ${ }^{*}, \dagger$, Lisa Delano-Wood ${ }^{\star}, \dagger$, Jody \\ Corey-Bloom $\uparrow, \ddagger$, and Mark W. Bondi ${ }^{*}, \dagger$ \\ * Department of Psychiatry, School of Medicine, University of California, San Diego 92093, USA \\ † Veterans Affairs San Diego Healthcare System, San Diego 92161, USA \\ ‡ Department of Neurosciences, School of Medicine, University of California, San Diego 92093, USA
}

\begin{abstract}
The original conceptualization of mild cognitive impairment (MCI) was primarily as an amnestic disorder representing an intermediate stage between normal aging and Alzheimer's dementia (AD). More recently, broader conceptualizations of MCI have emerged that also encompass cognitive domains other than memory. These characterizations delineate clinical subtypes that commonly include amnestic and non-amnestic forms, and that involve single and multiple cognitive domains. With the advent of these broader classifications, more specific information is emerging regarding the neuropsychological presentation of individuals with MCI, risk for dementia associated with different subtypes of MCI, and neuropathologic substrates connected to the clinical subtypes. This review provides an overview of this burgeoning literature specific to clinical subtypes of MCI. Focus is primarily on neuropsychological and structural neuroimaging findings specific to clinical subtypes of MCI as well as the issue of daily functioning. Although investigations of non-amnestic subtypes using advanced neuroimaging techniques and clinical trials are quite limited, we briefly review these topics in MCI because these data provide a framework for future investigations specifically examining additional clinical subtypes of MCI. Finally, the review comments on select methodological issues involved in studying this heterogeneous population, and future directions to continue to improve our understanding of $\mathrm{MCI}$ and its clinical subtypes are offered.
\end{abstract}

\section{Introduction}

Mild cognitive impairment (MCI) is a clinical construct that describes individuals with mildly impaired performance on objective neuropsychological tests but relatively intact global cognition and daily functioning (Petersen et al., 1999, 2001). MCI has been validated as qualitatively different from both normal aging and dementia (Petersen, 2004; Smith and Ivnik, 2003) and is a risk factor for the development of dementia. Because of its potential importance for early identification and intervention in those at risk for the development of dementia, the concept of MCI has received considerable research attention. However, the definition has evolved considerably over time. As originally proposed by Petersen and colleagues, MCI was characterized primarily as an amnestic disorder that represented an intermediate stage between normal aging and Alzheimer's dementia (AD) (Petersen et al., 1999). More recently, broader conceptualizations of MCI have emerged that also encompass cognitive domains other than memory (Petersen and Morris, 2005; Petersen et al., 2001). These characterizations delineate clinical subtypes that commonly include amnestic and non-amnestic forms, and that involve single and multiple cognitive domains (Manly et al., 2005; Petersen and Morris, 2005; Petersen et al., 2001; Tabert et al., 2006) against the backdrop of intact daily functioning. With the 
advent of these broader classifications schemes, more specific information is emerging regarding the neuropsychological presentation of individuals with MCI, risk for dementia associated with different subtypes of MCI, daily functioning, and neuropathologic substrates connected to the clinical subtypes. The aim of this review is to provide an overview of neuropsychological, neuroimaging, functional, and treatment findings specific to clinical subtypes of MCI. In addition, methodological issues involved in studying this heterogeneous population and future directions to continue to improve our understanding of MCI and its clinical subtypes will also be highlighted and discussed.

\section{Neuropsychological Presentation}

It is certainly of great interest to determine factors placing individuals at highest risk for development of dementia so as to target them for early intervention. To this end, a better understanding of who is at risk for developing MCI may be an important first step. Presently, there are limited data about risk factors that correspond to conversion from cognitively normal to specific clinical subtypes of MCI; although, the existing evidence suggests that advancing age and lower education levels do place individuals at higher risk for MCI (Kryscio et al., 2006), particularly for non-amnestic subtypes (Bickel et al., 2006; Fischer et al., 2007). For example, individuals with less than 9 years of education have an increased likelihood of isolated language and visuospatial deficits, as well as multiple domain amnestic MCI, whereas higher education was associated with increased chance of having isolated memory and executive impairments (Manly et al., 2005). The presence of the apolipoprotein epsilon 4 allele (APOE ع4) seems to more strongly influence transitions from normal cognition to amnestic MCI and influence conversion to multi-domain MCI to a lesser degree (Kryscio et al., 2006).

More research attention has been paid to the neuropsychological presentation of MCI and use of neuropsychological testing to delineate distinct clinical subtypes (e.g., amnestic vs nonamnestic and single domain vs multiple domain). Even when stratified into clinical subtypes, MCI is still a heterogeneous concept. Complicating factors include widely differing neuropsychological tests and diagnostic criteria used across studies in arriving at the MCI classifications as well as inconsistency in how clinical subtypes are assigned. While Petersen advocated for four subtypes, including single and multiple domain amnestic MCI and single and multiple domain non-amnestic MCI, non-amnestic subtypes continue to receive less attention in the literature and multiple domain classifications often do not separate amnestic from non-amnestic presentations. Despite these challenges, some converging evidence about the presentation of distinct MCI subtypes is emerging.

Generally, multi-domain presentations seem to be more common than purely amnestic presentations (Alexopoulos et al., 2006; Alladi et al., 2006; Lopez et al., 2003; Manly et al., 2005; Rasquin et al., 2005); although, some studies have identified single domains that are more common than multi-domains, and the non-amnestic type is as frequent as the amnestic (Busse et al., 2006; Palmer et al., 2008). Multi-domain MCI (mMCI) is the most common subtype in both stroke and memory clinic samples (Rasquin et al., 2005). The prevalence of single domain amnestic MCI (aMCI) ranges from 0.5 to 8\% (Bickel et al., 2006; Das et al., 2007; Di Carlo et al., 2007; Jungwirth et al., 2005; Lopez et al., 2003), mMCI ranges from 0.5 to 16\% (Bickel et al., 2006; Busse et al., 2003; Das et al., 2007; Di Carlo et al., 2007; Jungwirth et al., 2005; Lopez et al., 2003), and single domain non-amnestic MCI ranges from approximately 3 to 15\% (Bickel et al., 2006; Busse et al., 2003; Di Carlo et al., 2007). There is only limited information on prevalence rates of multi-domain non-amnestic MCI, in which one group has identified less than a 5\% prevalence rate (Bickel et al., 2006). In addition to vastly different methodological approaches to defining MCI, the prevalence rates also differ due to varying sample origins, with hospital samples having generally higher prevalence rates across subtypes than community samples (Bickel et al., 2006; Busse et al., 2003). Presence of 
the APOE $\varepsilon 4$ allele may also contribute to differing prevalence rates. Amnestic MCI presentations have a higher proportion of individuals with the apolipoprotein $\varepsilon 4$ allele as compared to non-amnestic groups (Gabryelewicz et al., 2007; Manly et al., 2005; Whitwell et al., 2007). Thus, if genetic risk is not determined, samples are likely to have differing proportions of individuals with the $\varepsilon 4$ allele and, therefore, different prevalence rates.

Certainly the prevalence rates are impacted by the definition of MCI applied by each study. Basing amnestic subtype diagnoses on the presence of either verbal and/or visual memory deficits results in a larger proportion of individuals identified as amnestic MCI than just relying on verbal memory alone (Alladi et al., 2006). Varying the cutoff score for defining impairment also alters diagnostic outcomes by up to $12 \%$; use of a more stringent statistical cutoff for impairment (1.5-2.0 SD below normative expectations) increases positive predictive power compared to lower cut points (Busse et al., 2003); although, a more liberal cutoff for impairment has been shown to have higher sensitivity and specificity for future development of dementia (Busse et al., 2006). These sensitivity and specificity determinations are problematical; however, as they are based on a quite limited neuropsychological assessment.

Aside from the use in diagnosis and determination of objective cognitive deficits in MCI, additional neuropsychological findings may help differentiate MCI subtypes. Perhaps not surprisingly, Lopez and colleagues (2006) found that, compared to aMCI and normal cognition, mMCI was characterized by poorer language, psychomotor speed, fine motor control, and visuoconstructional functioning. What is of note is that, although the mMCI group had memory deficits, they were to a lesser degree than the deficits noted in the aMCI group (Lopez et al., 2006). A substantial minority of the MCI cases did not have any memory impairment (Lopez et al., 2006), further emphasizing that examining only amnestic subtypes fails to capture the full spectrum of possible cognitive declines associated with MCI.

Spatial navigation skills of those with multi-domain amnestic MCI tend to be more similar to spatial navigation skills of $\mathrm{AD}$ patients than to non-amnestic MCI subtype groups, with both the AD and multi-domain amnestic MCI groups impaired on virtually all portions of a spatial navigation task (Hort et al., 2007). However, the multi-domain amnestic MCI group was generally more impaired than other groups across all neuropsychological tests, so it is not clear that these spatial navigation difficulties occurred in isolation from the other impaired functions. Visuospatial skills specific to facial emotional processing have also been found to be intact in those with single domain amnestic MCI but impaired in those with multi-domain amnestic MCI, particularly in facial affect discrimination (Teng et al., 2007).

\section{Stability of Diagnosis}

Multiple studies indicate that not all individuals diagnosed with MCI will decline and progress to a dementia diagnosis. In fact, a proportion of individuals appear to "improve" over time such that, at follow up, those initially identified as MCI are later categorized as cognitively normal. Anywhere from 20 (Fischer et al., 2007) to 40\% (Bickel et al., 2006) of those with MCI appear to revert to the normal range upon retesting. Single domain classifications appear particularly susceptible to this instability, with single domain non-amnestic subtype often exhibiting the least stability over time (Bickel et al., 2006; Busse et al., 2006; Fischer et al., 2007; Jak et $a l ., 2007)$. For example, one study reported that $50 \%$ of those with single domain MCI were normal upon later retesting whereas only $12 \%$ of those with multi-domain MCI "recovered" (Bickel et al., 2006). Additional sources of instability in the MCI diagnosis can manifest via individuals changing MCI subtypes over time. Approximately $6 \%$ of those with MCI at baseline changed subtypes at follow up (Fischer et al., 2007; Jak et al., 2007). In contrast, over a 3-year interval, Zanetti and colleagues (2006) identified a more anticipated 
trajectory of their MCI cohort; all MCI subtypes either converted to dementia (about a quarter) or retained their MCI status (Zanetti et al., 2006).

\section{Conversion to Dementia}

Perhaps the largest amount of information exists on likelihood of conversion to dementia from various MCI clinical subtypes. Some evidence suggests that those with multi-domain amnestic MCI appear to be at greatest risk for future dementia (Di Carlo et al., 2007; Palmer et al., 2008; Tabert et al., 2006), whereas others indicate that amnestic MCI places one at highest risk for conversion to dementia (Ravaglia et al., 2006; Yaffe et al., 2006). Amnestic MCI subtypes do seem to impart significant risk for future development of AD while multi-domain presentations may be more common in those who eventually develop vascular dementia (Fischer et al., 2007; Rasquin et al., 2005; Yaffe et al., 2006; Zanetti et al., 2006). Yaffe and colleagues (2006) found that, of those who progressed to $\mathrm{AD}, 76 \%$ were initially diagnosed with aMCI, $11 \%$ initially presented with single domain non-amnestic MCI, and $13 \%$ were initially identified as mMCI (Yaffe et al., 2006). Conversely, of those who progressed to vascular dementia, 50\% were initially diagnosed with aMCI, $8 \%$ had single domain nonamnestic MCI, and 42\% had mMCI (Yaffe et al., 2006). Rozzini and colleagues (2007) reported that, in a group of amnestic MCI individuals, poor global cognitive performance at baseline and worsening executive functioning, but not worsening memory performance, were associated with conversion to $\mathrm{AD}$ over a 1-year follow-up period. Those with non-amnestic multiple domain subtype appear more likely to convert to a non-AD dementia (Busse $e t$ al., 2006), with the single domain non-amnestic MCI at particular risk to progress to a frontal dementia syndrome (Yaffe et al., 2006). There are reports, however, in which detailed information about MCI subtypes does not add significant benefit in determining who may be at greatest risk for conversion to dementia (Maioli et al., 2007; Ravaglia et al., 2006; Rountree et al., 2007).

\section{V. $\mathrm{MCl}$ and Health Variables}

Understanding any additional health factors that may be more prevalent in distinct MCI subtypes is also noteworthy as a way to further delineate risk profiles. For example, cardiovascular risk factors, presence of the apolipoprotein $\varepsilon 4$ allele, mood symptoms, and parkinsonian symptoms have all been investigated in MCI subtypes. Recent research has shown that multi-domain or non-amnestic MCI subtypes may be more likely to have cardiovascular risk factors than either those with single domain amnestic presentations or those without MCI (Di Carlo et al., 2007; Zanetti et al., 2006). Mariani and colleagues (2007) found that those with single domain non-amnestic MCI had a higher frequency of ischemic heart disease, transient ischemic attack (TIA) or stroke, a higher Hachinski ischemic score, and more whitematter lesions on MRI compared to aMCI. Further, multi-domain and single domain amnestic subjects exhibited similar clinical characteristics; although, the multi-domain amnestic subtype did have a greater history of TIA/stroke (Mariani et al., 2007). Amnestic MCI groups showed a higher prevalence of diabetes than controls whereas participants with non-amnestic MCI were more likely to have hypertension than were controls (Verghese et al., 2008).

In contrast, Debette et al. (2007) found that white matter changes may play a role in cognitive decline in MCI as a whole, but they do not appear to be specific to either amnestic or nonamnestic clinical subtypes. Because their amnestic and non-amnestic characterizations were multi-domain, the authors found the rate of cognitive decline and the presence of periventricular hyperintensities was more prominent in those with baseline executive dysfunction (Debette $e t$ al., 2007).

Mood symptoms also appear to be more common in those with multi-domain MCI relative to those with single domain amnestic presentations (Gabryelewicz et al., 2007; Zanetti et al., 
2006). Additionally, higher depression ratings are linked to those whose cognitive symptoms progressed over the follow-up period (but not to the point of dementia) and to those who converted from MCI to dementia as compared to the stable group (Gabryelewicz et al., 2007). Hallucinations and sleep disorders are more common in the non-amnestic than amnestic subtype (Rozzini et al., 2008). Others have found, however, that mood symptoms are increased in MCI cohorts as compared to cognitively normal samples, but with no significant differences in mood symptoms emerging between specific MCI subtypes (Palmer et al., 2007; Rozzini et al., 2008).

Other health factors seem to be associated with MCI in general but are not necessarily specific to amnestic or non-amnestic presentations. For example, lower serum folate levels (Maioli et al., 2007; Ravaglia et al., 2006), history of atrial fibrillation (Ravaglia et al., 2006), and higher serum HDL levels (Maioli et al., 2007) contribute to increased likelihood of conversion from MCI to dementia, regardless of MCI subtype. Odor identification skills of MCI participants also fall in between those of $\mathrm{AD}$ and healthy control groups, and MCI subtypes did not differ on smell identification performances (Westervelt et al., 2008).

Most conceptualizations of MCI exclude Parkinson's disease, given that the historical conceptualization has been of MCI as a precursor to Alzheimer's disease or other nonParkinsonian dementias. In addition, the motor symptoms associated with Parkinson's disease often produce demonstrable changes in a person's activities of daily living (ADL), which confound its utility in the classification of MCI. Recently, however, there have been efforts to characterize the transitional period between normal cognitive function and dementia in Parkinson's disease (PD). The expanded clinical subtypes are particularly relevant to this effort given the difference in presentation between dementias of different origins. Data suggest that MCI in those with Parkinson's is predictive of future dementia in much the same way that it is for individuals with MCI without co-morbid PD. Non-amnestic subtypes are particularly prevalent in Parkinson's disease and the presence of MCI in individuals with Parkinson's disease does substantially raise one's risk of developing dementia as compared to those with $\mathrm{PD}$ and normal cognition (Janvin et al., 2006). In contrast to AD, conclusions about amnestic subtypes of PD MCI are more difficult to draw given the low prevalence of this subtype. Boyle et al. (2005) found that those individuals with MCI had higher levels of parkinsonian symptoms (though not Parkinson's disease) than those who were cognitively normal. Verghese et al. (2008) found greater gait abnormalities in those with aMCI as compared to those with nonamnestic MCI or controls while others have noted that non-amnestic subtypes have higher rates of gait dysfunction than amnestic MCI (Boyle et al., 2005). Those with mMCI may present with more extra-pyramidal features than those with aMCI (Zanetti et al., 2006).

\section{Daily Functioning and $\mathrm{MCl}$}

Embedded in the controversy surrounding the establishment of specific diagnostic criteria for MCI, there is much debate regarding whether impairment in everyday activities should be included as a criterion. In its initial conceptualization, MCI guidelines required that functional abilities remain intact (Petersen et al., 1999) as this specific criterion helped distinguish MCI from dementia. However, as Farias et al. (2006) note, cognitive and functional deterioration clearly occurs over the course of MCI since such change eventually leads to conversion to dementia for many individuals with MCI (e.g., Bruscoli and Lovestone, 2004; Petersen et $a l ., 1999)$. In light of this accumulating evidence, an international working group proposed modified criteria for MCI, which includes "preserved basic activities of daily living" and "minimal impairment in complex instrumental functions" (Winblad et al., 2004, p. 243). Similarly, participants of the International Psychogeriatric Association Expert Conference on MCI did not require intact ADL/instrumental activities of daily living (IADL) as a criterion but, instead, defined MCI as "a syndrome defined as cognitive decline greater than expected 
for an individual's age and education level but that does not interfere notably with activities of daily life" (Gauthier et al., 2006, p. 1262).

Emerging information about the functional status of those with distinct clinical subtypes of MCI may shed additional light on the continuum of functional abilities spanning normal cognition to dementia. Supporting the notion that functional decline occurs on a continuum, several groups have reported that IADL decrements in MCI are intermediate to the subtle declines associated with normal aging and the frank impairments required for a dementia diagnosis (Farias et al., 2006; Giovanetti et al., 2008; Griffith et al., 2003; Peres et al., 2006).

Published reports comparing ADL and IADL performance between MCI individuals and their cognitively normal counterparts have demonstrated that those with MCI show greater IADL changes in areas including shopping, managing medications, and handling finances (Mariani et al., 2008). In a study examining IADL performance across different MCI subtypes, Tam $e t$ al. (2007) reported that individuals with multiple domain MCI demonstrated impairment in IADL relative to both amnestic MCI participants and cognitively normal older adults. Corroborating this finding, Zanetti and colleagues (2006) demonstrated that individuals with multi-domain MCI performed more poorly on measures of ADL and IADL relative to amnestic MCI participants. In contrast, Farias and colleagues (2006) demonstrated that the MCI with memory impairment group showed somewhat more functional change relative to their MCI peers with no memory impairment and cognitively normal older adults. Similarly, Wadley and colleagues (2007) reported that amnestic, non-amnestic, and multiple domain MCI subgroups all demonstrated greater difficulty with IADL performance compared to the cognitively normal group. However, unlike the other two MCI groups, individuals with non-amnestic MCI did not differ from the cognitively normal participants in terms of IADL performance. Notably, at least one study (Boeve et al., 2003) did not find differences between amnestic MCI individuals and cognitively normal older adults in terms of functional abilities. However, it should be noted that the MCI participants who were included in this study were characterized as MCI based, in part, on intact ADL.

Farias and colleagues (2006) administered self-report and informant-based versions of the Daily Function Questionnaire (DFQ) and calculated a difference score by subtracting patients' DFQ from informants' DFQ. DFQ difference scores, which indicate a lack of awareness of deficits, were greater in demented individuals relative to their cognitively normal counterparts and MCI subtype groups (i.e., MCI with memory impairment and MCI without memory impairment). However, the difference scores did not differ between MCI groups and cognitively normal older adults. Based on these findings, Farias and colleagues (2006) argued that individuals with MCI do not underestimate functional changes as is often the case for demented individuals.

What seems clear from the above discussion is that changes in ADL and IADL are not uncommon across the spectrum of MCI; although, it remains to be determined whether many of these changes would be conceptualized as frank "deficits" or "impairments." Comparing the ADL and IADL changes of MCI to overt dementia groups would be helpful in determining cutoff criteria for impairment, or administering performance-based ADL and IADL measures with normative reference standards would also help to delineate whether such changes represent impairment.

\section{Neuroimaging}

\section{A. Structural MRI}

In determining the clinical viability of the various clinical subtypes, many would assert that different subtypes should have distinct neuropathology or different courses of change in brain 
integrity. Certainly, structural neuroimaging provides a non-invasive way to begin to examine brain changes associated with MCI, and there is emerging evidence to support distinct neuropathological profiles in clinical subtypes of MCI. Whitwell et al. (2007) found that those with amnestic presentations (single or multiple domain) had greater gray matter atrophy in medial and inferior temporal lobes compared to controls. Those with multi-domain amnestic MCI additionally showed loss in posterior temporal lobe, parietal association cortex, posterior cingulate, anterior insula, and the medial frontal lobe, a pattern of atrophy similar to that found in AD (Whitwell et al., 2007). In support of this finding, Seo and colleagues (2007) reported that those diagnosed with single domain amnestic MCI showed cortical thinning in left medial temporal lobe (MTL) only, whereas those identified as multi-domain amnestic MCI showed cortical thinning in the left MTL, precuneus, and anterior and inferior basal temporal, insular, and temporal association cortices. The precuneus atrophy may be responsible for additional cognitive impairments present in the multi-domain MCI subtype and may suggest that the multi-domain presentations are a progression from single domain presentations since the areas of thinning noted in the multi-domain subtype encompassed all those in the single domain subtype and the extent of MTL atrophy was greater in the multi-domain versus the single domain subtype (Seo et al., 2007).

In contrast, Becker and colleagues (2006) did not support the multi-domain subtype as the more advanced, transitional state between normal cognition and AD. They found that hippocampal volumes in those with multi-domain MCI were not statistically different from those of controls, but were significantly larger than both the amnestic MCI and AD groups (Becker et al., 2006).Bell McGinty and colleagues (2005) found that the amnestic MCI group had greater volume loss in left entorhinal cortex and inferior parietal lobe as compared with multi-domain MCI. However, the multi-domain MCI group may exhibit their neuropathological changes in other areas, namely by smaller right inferior frontal gyrus, right middle temporal gyrus, and bilateral superior temporal gyrus as compared to amnestic MCI (Becker et al., 2006).

Although the data are conflicting as to whether multi-domain subtypes necessarily have more extensive brain changes than single domain subtypes, current research does support the idea that different clinical subtypes of MCI have distinct neuropathology. Taken together, the available evidence suggests that those individuals with a more focal memory presentation have greater involvement of mesial temporal structures while those with more widespread deficits had greater involvement of association areas. Distinct MCI subtypes may represent different etiological paths to dementia, but the small sample sizes available in most of the imaging studies to date make conclusions tentative at best.

Other advanced imaging techniques hold promise to further clarify the nature and extent of brain changes associated with distinct clinical MCI subtypes though, to date, use of techniques such as functional magnetic resonance imaging (FMRI) and diffusion tensor imaging (DTI) has focused globally on MCI or on amnestic MCI, without significant investigation of nonamnestic subtypes. An overview of the use of these imaging techniques in MCI is provided, nonetheless, as this preliminary work is an essential framework for future examinations of clinical MCI subtypes.

\section{B. Diffusion Tensor Imaging}

A growing body of research suggests that white matter pathology contributes to age-related cognitive impairment and possibly potentiates the development of dementia (Raz and Rodrigue, 2006; Sullivan and Pfefferbaum, 2006). Although studies have generally shown white matter changes to be accelerated and more severe in AD (Pfefferbaum et al., 2000; Rose et al., 2006; Takahashi et al., 2002), to date, few studies have employed DTI to examine early white matter changes in older adults with MCI with exceptionally limited focus on DTI-derived white matter changes in specific MCI clinical subtypes. Several studies have shown reduced 
fractional anisotropy (FA), a proxy of white matter integrity, in the posterior cingulum fibers, and this relationship seems to be stronger in the left versus right hemisphere (Fellgiebel $e t$ al., 2005; Medina et al., 2006; Rose et al., 2006; Zhang et al., 2007). Rose et al. (2006) demonstrated increased diffusivity in the entorhinal and parieto-occipital cortices, and decreased FA in the limbic parahippocampal white matter in patients with MCI. Moreover, Kantarci et al. (2005) was among the first to show that increased mean diffusivity of the hippocampus in amnestic MCI predicted future progression to dementia.

Several studies have shown decreased integrity in the posterior region of the corpus callosum (i.e., splenium) in those with MCI (Cho et al., 2008; Delano-Wood et al., 2007; Ukmar et al., 2008), an area which is particularly sensitive to degenerative processes (Naggara et al., 2006; Rose et al., 2000; Takahashi et al., 2002). Although some studies have shown changes in the frontal white matter of MCI patients (Bozzali et al., 2002; Medina et al., 2006; Naggara et al., 2006), other studies have not identified any differences (Delano-Wood et al., 2007; Head et al., 2004; Medina et al., 2006; Ukmar et al., 2008). Although data are limited, results suggest a pattern of retrogenesis (Bartzokis, 2004), by which microstructural changes first occur in late-myelinating regions, spreading to early-myelinating regions only after the disease process has progressed beyond a particular threshold, which may initially manifest itself with the onset of MCI.

\section{Functional MRI}

Evidence to date indicates that functional brain decline precedes structural decline in prodromal dementia, including adults with MCI. Therefore, functional neuroimaging techniques may offer the unique ability to detect early functional brain changes in at-risk adults and identify the neurophysiological markers that best predict dementia conversion.

Given that AD neuropathology preferentially targets the MTL early in the course of the disease, thereby resulting in the hallmark episodic memory decline, and amnestic $\mathrm{MCI}$ is thought to represent prodromal $\mathrm{AD}$, the majority of FMRI studies of MCI involve memory processing (particularly encoding) in amnestic samples. No known FMRI studies have been published focusing on other clinical subtypes of MCI. While several studies demonstrate increased blood oxygen level dependent (BOLD) response in the MTL (Dickerson et al., 2004, 2005; Hamalainen et al., 2007; Kircher et al., 2007; Sperling, 2007), others report decreased MTL activity in MCI (Johnson et al., 2006; Machulda et al., 2003; Mandzia et al., 2009). These discrepant findings have been interpreted as reflecting bimodal functional activity whereby less impaired MCI subjects show increased BOLD response in the hippocampus corresponding to a slight or moderate neuronal dysfunction, and more impaired MCI subjects demonstrate decreased BOLD response-similar to the levels observed in mild AD patients-as the cortical neuronal networks become more severely impaired with greater disease progression (Celone et al., 2006; Dickerson et al., 2004, 2005; Hamalainen et al., 2007; Johnson et al., 2006; Machulda et al., 2003; Masdeu et al., 2005; Petrella et al., 2007a). However, this interpretation is primarily derived from cross-sectional studies and can only adequately be tested with longitudinal designs.

Few longitudinal FMRI studies of MCI have been reported. Although these studies are often limited by small sample sizes, they demonstrate promise for the use of FMRI to detect early $\mathrm{AD}$. Those MCI patients who converted to $\mathrm{AD}$ showed a stronger relationship between brain activity in the left superior parietal lobe and the left precuneus during an angle discrimination task in the context of comparable performance (Vannini et al., 2007). Similarly, despite equivalent memory performance, Dickerson et al. (2004) reported that MCI patients who subsequently declined during a 2.5-year follow-up period demonstrated increased right parahippocampal gyrus activity during picture encoding. In a more recent study, the same research group reported increased hippocampal activation predicted greater degree and rate of 
cognitive decline during a 6-year follow-up period, even after controlling for baseline level of impairment (Miller et al., 2008).

Mandzia et al. (2009) reported that MTL activation during recognition was positively correlated with behavioral performance. However, unlike their healthy peers, MCI adults did not show a strong relationship between MTL activity during picture encoding and subsequent retrieval success, highlighting the complexity of the relationship between BOLD signal and effectiveness of encoding strategies. In contrast, Johnson et al. (2006) found reduced BOLD signal change in the right hippocampus during picture encoding and in the posterior cingulate during recognition of learned items in an amnestic MCI group despite comparable performance to their healthy peers. However, when activation corresponding only to successfully learned words was examined, an increase in hippocampal activity was seen, suggesting that an increase in MTL activity may support successful memory encoding (Kircher et al., 2007). Similarly, a positive correlation between extent of parahippocampal and hippocampal activation and memory performance was found in MCI but, in a paradoxical fashion, greater clinical impairment, was also associated with recruitment of a larger region of the right parahippocampal gyrus during encoding (Dickerson et al., 2004). Data from Johnson et al. (2004) provided further evidence for hippocampal dysfunction in MCI, suggesting that adults with MCI do not habituate to increasingly familiar items in the same manner as healthy older adults who show expected reductions in BOLD response to repeated items over time.

Despite the prevalence of studies examining medial temporal cortex function supporting memory, other cortical areas have also been implicated in MCI. For example, a reduction in functional activity in the posterior cingulate cortex (PCC) during recognition and episodic retrieval of previously learned line drawings (Johnson et al., 2006) and object working memory (Yetkin et al., 2006), but not during self-appraisal (Ries et al., 2007), has implicated this region in the memory retrieval difficulty seen in amnestic MCI. The degradation of PCC functioning in $\mathrm{MCI}$ is not surprising given that PET metabolic alterations in the temporoparietal cortices and in the posterior cingulate have been reported in MCI and AD (Desgranges et al., 1998; Matsuda, 2001; Reiman et al., 1996) as well as in nondemented young and middle-aged adults at genetic risk for AD (Petrella et al., 2007b; Reiman et al., 1996, 2004, 2005; Wolf et al., 2003). Similarly, dedifferentiation in the retrosplenial cortex during the retrieval of recent versus remote autobiographical memories and during episodic versus semantic memory retrieval has been reported in amnestic MCI (Poettrich et al., 2009), further implicating the medial posterior cortex in MCI. Additionally, the neural substrates of visual working memory (Yetkin et al., 2006), self-appraisal (Ries et al., 2007), and emotional working memory (Dohnel et al., 2008) in MCI have also been examined, and generally implicate a greater number of cortical regions. However, results are varied and highlight the need for greater attention to other cognitive processes in $\mathrm{MCI}$ in order to more fully understand changes in cortical functioning that may signal impending cognitive decline.

\section{Treatment}

One motivation to better understand the heterogeneous concept of MCI and the risk it imparts for future development of dementia is to provide early interventions that could halt or at least slow progression of symptoms. To date, unfortunately, there are no FDA-approved therapies for MCI. Further, aMCI has received all the attention with regard to treatment trials with no trials investigating other distinct clinical subtypes of MCI. Of the existing treatment trials in MCI, most have used a "progression to AD" design with the focus on slowing cognitive decline and delaying conversion to AD. As a whole, the trials have been disappointing with one possible exception, the Alzheimer's Disease Cooperative Study (ADCS)-sponsored trial (Petersen et al., 2005) (see Table I). 
The ADCS-sponsored study of Vitamin E and donepezil for MCI involved 769 subjects at 69 centers in the US and Canada over 3 years. There were three treatment arms: Vitamin E 2000 IU/day, donepezil $10 \mathrm{mg} / \mathrm{day}$, and placebo. The primary trial endpoint was conversion to AD. Although conversion to AD favored donepezil at 1 year, there were no differences among groups with regard to conversion to AD at 3 years. However, possession of the APOE $\varepsilon 4$ allele was noted to be associated with a threefold greater risk of conversion from aMCI to dementia and, thus, clearly an important predictor of progression. When the authors looked at the progression to $\mathrm{AD}$ for $\mathrm{APOE} \varepsilon 4$ positive participants by treatment group, they found that the effect of donepezil was greater in $\varepsilon 4$ positive individuals and persisted for 2 years. While neither of the two active arms reduced the risk of progressing to $\mathrm{AD}$ over the entire 36 months, donepezil reduced the risk of progression to AD for the first 12 months in all subjects and up to 24 months in those who were positive for the APOE $\varepsilon 4$ allele. No treatment effect was noted for Vitamin E.

Other treatment trials have been less promising for halting conversion from MCI to dementia over time. A large trial of rivastigmine, an acetylcholinesterase inhibitor, was a double blind, placebo-controlled trial of 1018 patients that had many of the same features as the ADCS trial, but was conducted in 14 countries using multiple languages and translations of the neuropsychological instruments (Feldman et al., 2007). At baseline, arms were not well matched with regard to frequency of APOE $\varepsilon 4$ genotype, which was $46 \%$ in the placebo arm but only $37 \%$ in the rivastigmine arm. The study also had a lower conversion rate than expected and had to be extended to 4 years; over that time, $21.4 \%$ of placebo treated, but only $17.3 \%$ of rivastigmine treated, subjects progressed to $\mathrm{AD}$. Although rivastigmine was favored, the results were not statistically significant, and secondary assessments were also not significant.

Investigation of the efficacy of another acetylcholinesterase inhibitor, galantamine, also failed to reveal a significant effect of galantamine on conversion to dementia in those with $\mathrm{MCI}$ in either of two trials (Winblad et al., 2008).

Finally, another large randomized, placebo-controlled, double-blind study examined the ability of the COX 2 inhibitor, rofecoxib, to delay disease progression in 1457 aMCI subjects (Thal et al., 2005). Once again, there was a lower than expected annual rate of conversion to AD. Conversion to $\mathrm{AD}$ actually favored placebo in this trial but the authors dismissed the significance of this finding because the secondary cognitive measures did not corroborate the primary outcome.

In hindsight, several important factors likely influenced the results of these studies, including, perhaps first and foremost, the variable rate of progression from aMCI to AD. Sources of this variability likely include subject heterogeneity, with regard to impairment level, culture, language, and APOE $\varepsilon 4$ carrier status, in addition to even simple differences in implementation of enrollment criteria. MCI patients may show increased awareness of, or lower tolerability for, adverse events, resulting in higher discontinuation rates. Our current outcome measures may be insensitive; for example, the conversion design dichotomizes a continuous variable and most of the currently used efficacy measures follow an AD trial model of decline. Rather than decline, however, MCI patients may show improvement on cognitive measures, no matter which treatment group they are assigned to, because of at least some preservation in their ability to learn. Future MCI trials may benefit from less heterogeneous recruitment with stricter entry criteria and enriched populations, more sensitive cognitive and global outcome measures that reflect subtle impairments in complex activities, novel imaging outcomes, and longer trials.

\section{Conclusions}

MCI remains a heterogeneous concept, though division of MCI into distinct clinical subtypes serves as a promising approach to better understanding $\mathrm{MCI}$ as a diagnostic entity and a risk 
factor for future cognitive decline. Evidence to date suggests that multi-domain amnestic presentations are more prevalent than either single domain amnestic or multi-domain nonamnestic presentations, though relatively little attention has been paid to the latter subtype. Converging neuropsychological, daily functioning, and neuroimaging data suggest that multiple domain presentations may place one at highest risk for future development of dementia. The current literature also supports that knowledge of subtypes of MCI informs the risk for future development of different types of dementia.

Though knowledge of MCI subtypes appears helpful in predicting risk of conversion to dementia, there remains a significant minority of individuals with MCI, particularly single domain subtype that may revert to normal cognition when followed over time. This instability in diagnosis as well as the varying prevalence rates, rates of conversion to dementia, and general oft-conflicting results in the literature, are likely due to ongoing challenges in operationalizing the diagnostic criteria for MCI. The methods for documenting objective neuropsychological impairment tend to be a particularly variable and ill-defined aspect of the MCI diagnostic process across studies (Portet et al., 2006). There is little consensus about what neuropsychological tests (or how many) should be used to document objective cognitive impairment, what level of performance is considered cognitively impaired, how diagnostic criteria for different clinical subtypes of MCI are applied, what constitutes intact daily functioning, or about whether or not functional abilities should be included in the diagnostic decision-making regarding MCI. As this review highlights, the variable results in the current MCI literature clearly illustrate the importance of (a) understanding the criteria used to identify cognitive impairment in making the MCI diagnosis, (b) the value of using comprehensive neuropsychological assessment when diagnosing MCI subtypes, and (c) point to the need for further exploration of MCI subtypes, particularly non-amnestic presentations. Investigations and interventions targeting only amnestic subtypes are potentially missing a sizable number of individuals at risk.

Neuroimaging holds promise as a technique to better understand differences between distinct MCI subtypes although all of the above-mentioned methodological challenges together with small sample sizes and very limited attention paid to non-amnestic subtypes make drawing firm conclusions from the existing imaging literature challenging. To date, data do seem to support distinct neuropathology in the different clinical subtypes of MCI. However, there is still much overlap in structural imaging profiles and conflicting evidence making conclusions tentative at best. Advanced imaging techniques, such as DTI and functional MRI hold promise for detecting microstructural white matter damage or altered activation patterns in older adults prior to the manifestation of the full dementia syndrome. This early identification would identify the group in whom targeted therapies will likely have the greatest clinical impact (see Fagan et al., 2005, for discussion). Overall, results from recent DTI studies indicate that white matter changes are evident in at-risk older adults and further validate the use of DTI to capture subtle, early white matter changes before significant atrophy is present. However, to date, very few studies have employed DTI in older adults with MCI, and even fewer have investigated the relationship between clinical subtype of MCI and white matter integrity.

Similarly, although FMRI techniques may prove to be instrumental in the early detection of $\mathrm{AD}$, interpretation of current findings in MCI is complicated by various methodological differences between studies. In general, functional changes in the MTL and posterior medial cortex appear to signal cognitive decline and dementia conversion. However, discrepant results across studies may be due to differences in diagnostic classification of MCI adults. Specifically, although the majority of studies reviewed classified their patients as amnestic MCI, it is likely that the patient sample represented a more heterogeneous group that may reflect different underlying neural pathology. This highlights the need for future research aimed at integrating 
behavioral performance with measures of functional activity that directly compare different MCI subtypes with these sophisticated neuroimaging techniques.

Finally, results of intervention trials to halt the progression of MCI have generally been disappointing. Future trials are needed that address both amnestic and non-amnestic presentations, employ more stringent entry criteria, use more sensitive cognitive and global outcome measures that reflect subtle impairments in complex activities, and include novel imaging outcomes.

\section{Acknowledgments}

This work was supported by grants from the National Institutes of Health (K24 AG026431, R01 AG012674, and P50 AG05131), by Career Development Awards from the Department of Veterans Affairs, and by Investigator-Initiated and New Investigator Research Grants from the Alzheimer's Association. The authors gratefully acknowledge the assistance of staff, patients, and volunteers of the UCSD Alzheimer's Disease Research Center, and the UCSD Laboratory of Cognitive Imaging.

\section{References}

Alexopoulos P, Grimmer T, Perneczky R, Domes G, Kurz A. Progression to dementia in clinical subtypes of mild cognitive impairment. Dement Geriatr Cogn Disord 2006;22:27-34. [PubMed: 16679762]

Alladi S, Arnold R, Mitchell J, Nestor PJ, Hodges JR. Mild cognitive impairment: Applicability of research criteria in a memory clinic and characterization of cognitive profile. Psychol Med 2006;36:19.

Bartzokis G. Age-related myelin breakdown: A developmental model of cognitive decline and Alzheimer's disease. Neurobiol Aging 2004;25:5-18. [PubMed: 14675724]

Becker JT, Davis SW, Hayashi KM, Meltzer CC, Toga AW, Lopez OL, Thompson PM. Threedimensional patterns of hippocampal atrophy in mild cognitive impairment. Arch Neurol 2006;63:97101. [PubMed: 16401741]

Bell-McGinty S, Lopez OL, Meltzer CC, Scanlon JM, Whyte EM, DeKosky ST, et al. Differential cortical atrophy in subgroups of mild cognitive impairment. Arch Neurol 2005;62:1393-1397. [PubMed: 16157746]

Bickel H, Mösch E, Seigerschmidt E, Siemen M, Förstl H. Prevalence and persistence of mild cognitive impairment among elderly patients in general hospitals. Dement Geriatr Cogn Disord 2006;21:242250. [PubMed: 16465052]

Boeve B, McCormick J, Smith G, Ferman T, Rummans T, Carpenter T, Ivnik R, Kokmen E, Tangalos E, Edland S, Knopman D, Petersen R. Mild cognitive impairment in the oldest old. Neurology 2003;60:477-480. [PubMed: 12578930]

Boyle PA, Wilson RS, Aggarwal NT, Arvanitakis Z, Kelly J, Bienias JL, Bennett DA. Parkinsonian signs in subjects with mild cognitive impairment. Neurology 2005;65:1901-1906. [PubMed: 16380610]

Bozzali M, Falini A, Franceschi M, Cercignani M, Zuffi M, Scotti G, Comi G, Filippi M. White matter damage in Alzheimer's disease assessed in vivo using diffusion tensor magnetic resonance imaging. J Neurol Neurosurg Psychiatry 2002;72:742-746. [PubMed: 12023417]

Bruscoli M, Lovestone S. Is MCI really just early dementia? A systematic review of conversion studies. Int Psychogeriatr 2004;16:12-40.

Busse A, Bischkopf J, Riedel-Heller SG, Angermeyer MC. Subclassifications for mild cognitive impairment: Prevalence and predictive validity. Psychol Med 2003;33:1029-1038. [PubMed: 12946087]

Busse A, Hensel A, Guhne U, Angermeyer MC, Riedel-Heller SG. Mild cognitive impairment: Longterm course of four clinical subtypes. Neurology 2006;67:2176-2185. [PubMed: 17190940]

Celone KA, Calhoun VD, Dickerson BC, Atri A, Chua EF, Miller SL, DePeau K, Rentz DM, Selkoe DJ, Blacker D, Albert MS, Sperling RA. Alterations in memory networks in mild cognitive impairment and Alzheimer's disease: An independent component analysis. J Neurosci 2006;26:10222-10231. [PubMed: 17021177] 
Cho H, Dong WY, Shon YM, Kim BS, Kim YI, Choi YB, Lee KS, Shim YS, Yoon B, Kim W, Ahn KJ. Abnormal integrity of corticocortical tracts in mild cognitive impairment: A diffusion tensor imaging study. J Korean Med Sci 2008;23:477-483. [PubMed: 18583886]

Das SK, Bose P, Biswas A, Dutt A, Banerjee TK, Hazra A, Raut DK, Chaudhuri A, Roy T. An epidemiologic study of mild cognitive impairment in Kolkata, India. Neurology 2007;68:2019-2026. [PubMed: 17548552]

Debette S, Bombois S, Bruandet A, Delbeuck X, Lepoittevin S, Delmaire C, Leys D, Pasquier F. Subcortical hyperintensities are associated with cognitive decline in patients with mild cognitive impairment. Stroke 2007;38:2924-2930. [PubMed: 17885256]

Delano-Wood L, Jak AJ, Schweinsburg B, Wierenga C, Horne N, Salmon DP, Thal LJ, Frank LR, Bondi MW. Posterior white matter changes in MCI: Associations with cognition and stroke risk. J Int Neuropscyhol Assoc 2007;13:81.

Desgranges B, Baron JC, de la Sayette V, Petit-Taboue MC, Benali K, Landeau B, Lechevalier B, Eustache F. The neural substrates of memory systems impairment in Alzheimer's disease. A PET study of resting brain glucose utilization. Brain 1998;121(Pt 4):611-631. [PubMed: 9577389]

Di Carlo A, Lamassa M, Baldereschi M, Inzitari M, Scafato E, Farchi G, Inzitari D. CIND and MCI in the Italian elderly: Frequency, vascular risk factors, progression to dementia. Neurology 2007;68:1909-1916. [PubMed: 17536047]

Dickerson BC, Salat DH, Bates JF, Atiya M, Killiany RJ, Greve DN, Dale AM, Stern CE, Blacker D, Albert MS, Sperling RA. Medial temporal lobe function and structure in mild cognitive impairment. Ann Neurol 2004;56:27-35. [PubMed: 15236399]

Dickerson BC, Salat DH, Greve DN, Chua EF, Rand-Giovannetti E, Rentz DM, Bertram L, Mullin K, Tanzi RE, Blacker D, Albert MS, Sperling RA. Increased hippocampal activation in mild cognitive impairment compared to normal aging and AD. Neurology 2005;65:404-411. [PubMed: 16087905]

Dohnel K, Sommer M, Ibach B, Rothmayr C, Meinhardt J, Hajak G. Neural correlates of emotional working memory in patients with mild cognitive impairment. Neuropsychologia 2008;46:37-48. [PubMed: 17915264]

Fagan AM, Csernansky CA, Morris JC, Holtzman DM. The search for antecedent biomarkers of Alzheimer's disease. J Alzheimer's Dis 2005;8:347-358.

Farias ST, Mungas D, Reed BR, Harvey D, Cahn-Weiner D, DeCarli C. MCI is associated with deficits in everyday functioning. Alzheimer Dis Assoc Disord 2006;20:217-223. [PubMed: 17132965]

Feldman HH, Ferris S, Winblad B, Sfikas N, Mancione L, He Y, Tekin S, Burns A, Cummings J, del Ser T, Inzitari D, Orgogozo JM, et al. Effect of rivastigmine on delay to diagnosis of Alzheimer's disease from mild cognitive impairment: the InDDEx study. Lancet 2007;6:501-512.

Fellgiebel A, Muller MJ, Wille P, Dellani PR, Scheurich A, Schmidt LG, Stoeter P. Color-coded diffusion-tensor-imaging of posterior cingulate fiber tracts in mild cognitive impairment. Neurobiol Aging 2005;26:1193-1198. [PubMed: 15917103]

Fischer P, Jungwirth S, Zehetmayer S, Weissgram S, Hoenigschnabl S, Gelpi E, Krampla W, Tragl KH. Conversion from subtypes of mild cognitive impairment to Alzheimer dementia. Neurology 2007;68:288-291. [PubMed: 17242334]

Gabryelewicz T, Styczynska M, Luczywek E, Barczak A, Pfeffer A, Androsiuk W, ChodakowskaZebrowska M, Wasiak B, Peplonska B, Barcikowska M. The rate of conversion of mild cognitive impairment to dementia: Predictive role of depression. Int J Geriatr Psychiatry 2007;22:563-567. [PubMed: 17136705]

Gauthier S, Reisberg B, Zaudig M, Petersen RC, Ritchie K, Broich K, Belleville S, Brodaty H, Bennett D, Chertkow H, Cummings JL, de Leon M, et al. Mild cognitive impairment. The Lancet 2006;367:1262-1270.

Giovanetti T, Bettcher BM, Brennan L, Libon DJ, Burke M, Duey K, Nieves C, Wambach D. Characterization of everyday functioning in mild cognitive impairment: A direct assessment approach. Dement Geriatr Cogn Disord 2008;25:359-365. [PubMed: 18340108]

Griffith HR, Belue BS, Sicola A, Krzywanski S, Zamrini E, Harrell L, Marson DC. Impaired financial abilities in mild cognitive impairment: A direct assessment approach. Neurology 2003;60:449-457. [PubMed: 12578926] 
Hamalainen A, Pihlajamaki M, Tanila H, Hanninen T, Niskanen E, Tervo S, Karjalainen PA, Vanninen RL, Soininen H. Increased fMRI responses during encoding in mild cognitive impairment. Neurobiol Aging 2007;28:1889-1903. [PubMed: 16997428]

Head D, Buckner RL, Shimony JS, Williams LE, Akbudak E, Conturo E, McAvoy M, Morris JC, Snyder AZ. Differential vulnerability of anterior white matter in nondemented aging with minimal acceleration in dementia of the Alzheimer's type: Evidence form diffusion tensor imaging. Cerebral Cortex 2004;14:410-423. [PubMed: 15028645]

Hort J, Laczo J, Vyhnalek M, Bojar M, Bures J, Vlcek K. Spatial navigation deficit in amnesic mild cognitive impairment. Proc Natl Acad Sci USA 2007;104:4042-4047. [PubMed: 17360474]

Jak AJ, Corey-Bloom J, Bondi MW. Diagnostic characterization of MCI subtypes in a naturalistic sample. J Int Neuropscyhol Assoc 2007;13:4.

Janvin CC, Larsen JP, Aarsland D, Hugdahl K. Subtypes of mild cognitive impairment in Parkinson's disease: Progression to dementia. Mov Disord 2006;21:1343-1349. [PubMed: 16721732]

Johnson SC, Baxter LC, Susskind-Wilder L, Connor DJ, Sabbagh MN, Caselli RJ. Hippocampal adaptation to face repetition in healthy elderly and mild cognitive impairment. Neuropsychologia 2004;42:980-989. [PubMed: 14998712]

Johnson SC, Schmitz TW, Moritz CH, Meyerand ME, Rowley HA, Alexander AL, Hansen KW, Gleason CE, Carlsson CM, Ries ML, Asthana S, Chen K, et al. Activation of brain regions vulnerable to Alzheimer's disease: The effect of mild cognitive impairment. Neurobiol Aging 2006;27:1604-1612. [PubMed: 16226349]

Jungwirth S, Weissgram S, Zehetmayer S, Tragl KH, Fischer P. VITA: Subtypes of mild cognitive impairment in a community-based cohort at the age of 75 years. Int J Geriatr Psychiatry 2005;20:452458. [PubMed: 15852463]

Kantarci K, Petersen RC, Boeve BF, Knopman DS, Weigand SD, O'Brien PC, et al. DWI predicts future progression to Alzheimer disease in amnestic mild cognitive impairment. Neurology 2005;64:902904. [PubMed: 15753434]

Kircher TT, Weis S, Freymann K, Erb M, Jessen F, Grodd W, Heun R, Leube DT. Hippocampal activation in patients with mild cognitive impairment is necessary for successful memory encoding. $\mathrm{J}$ Neurol Neurosurg Psychiatry 2007;78:812-818. [PubMed: 17287238]

Kryscio RJ, Schmitt FA, Salazar JC, Mendiondo MS, Markesbery WR. Risk factors for transitions from normal to mild cognitive impairment and dementia. Neurology 2006;66:828-832. [PubMed: 16567698]

Lopez OL, Jagust WJ, DeKosky ST, Becker JT, Fitzpatrick A, Dulberg C, Breitner J, Lyketsos C, Jones B, Kawas C, Carlson M, Kuller LH. Prevalence and classification of mild cognitive impairment in the cardiovascular health study cognition study: Part 1. Arch Neurol 2003;60:1385-1389. [PubMed: 14568808]

Lopez OL, Becker JT, Jagust WJ, Fitzpatrick A, Carlson MC, DeKosky ST, Breitner J, Lyketsos CG, Jones B, Kawas C, Kuller LH. Neuropsychological characteristics of mild cognitive impairment subgroups. J Neurol Neurosurg Psychiatry 2006;77:159-165. [PubMed: 16103044]

Machulda MM, Ward HA, Borowski B, Gunter JL, Cha RH, O'Brien PC, Petersen RC, Boeve BF, Knopman D, Tang-Wai DF, Ivnik RJ, Smith GE, et al. Comparison of memory fMRI response among normal, MCI, and Alzheimer's patients. Neurology 2003;61:500-506. [PubMed: 12939424]

Maioli F, Coveri M, Pagni P, Chiandetti C, Marchetti C, Ciarrocchi R, Ruggero C, Nativio V, Onesti A, D'Anastasio C, Pedone V. Conversion of mild cognitive impairment to dementia in elderly subjects: A preliminary study in memory and cognitive disorder unit. Arch Gerontol Geriatr 2007;44(Suppl 1):233-241. [PubMed: 17317458]

Mandzia JL, McAndrews MP, Grady CL, Graham SJ, Black SE. Neural correlates of incidental memory in mild cognitive impairment: An fMRI study. Neurobiol Aging 2009;30:717-730. [PubMed: 17963998]

Manly JJ, Bell-McGinty S, Tang M, Schupf N, Stern Y, Mayeux R. Implementing diagnostic criteria and estimating frequency of mild cognitive impairment in an urban community. Arch Neurol 2005;62:1739-1746. [PubMed: 16286549] 
Mariani E, Monastero R, Ercolani S, Mangialasche F, Caputo M, Feliziani FT, Vitale DF, Senin U, Mecocci P. Vascular risk factors in mild cognitive impairment subytpes. Dement Geriatr Cogn Disord 2007;24:448-456. [PubMed: 17975314]

Mariani E, Monastero R, Ercolani S, Rinaldi P, Mangialasche F, Costanzi E, Vitale DF, Senin U, Mecocci P. Influence of comorbidity and cognitive status on instrumental activities of daily living in amnestic mild cognitive impairment: Results from the ReGAI project. Int J Geriatr Psychiatry 2008;23:523530. [PubMed: 18058828]

Masdeu JC, Zubieta JL, Arbizu J. Neuroimaging as a marker of the onset and progression of Alzheimer's disease. J Neurol Sci 2005;236:55-64. [PubMed: 15961110]

Matsuda H. Cerebral blood flow and metabolic abnormalities in Alzheimer's disease. Ann Nucl Med 2001;15:85-92. [PubMed: 11448080]

Medina D, deToledo-Morrell L, Urresta F, Gabrieli JDE, Moseley M, Fleischman D, Bennett DA, Leurgans S, Turner DA, Stebbins GT. White matter changes in mild cognitive impairment and AD: A diffusion tensor imaging study. Neurobiol Aging 2006;27:663-672. [PubMed: 16005548]

Miller SL, Fenstermacher E, Bates J, Blacker D, Sperling RA, Dickerson BC. Hippocampal activation in adults with mild cognitive impairment predicts subsequent cognitive decline. J Neurol Neurosurg Psychiatry 2008;79:630-635. [PubMed: 17846109]

Naggara O, Oppenheim C, Rieu D, Raoux N, Rodrigo S, Barba GD, Meder JF. Diffusion tensor imaging in early Alzheimer's disease. Psychiatry Res 2006;146:663-672.

Palmer K, Berger AK, Monastero B, Winblad B, Backman L, Fratiglioni L. Predictors of progression from mild cognitive impairment to Alzheimer disease. Neurology 2007;68:1596-1602. [PubMed: 17485646]

Palmer K, Backman L, Winblad B, Fratiglioni L. Mild cognitive impairment in the general population: Occurrence and progression to Alzheimer disease. Am J Geriatr Psychiatry 2008;16:603-611. [PubMed: 18591580]

Peres K, Chrysostome V, Fabrigoule C, Orgogozo JM, Dartigues JF, Barberger-Gateau P. Restriction in complex activities of daily living in MCI: Impact on outcome. Neurology 2006;67:461-466. [PubMed: 16894108]

Petersen RC. Mild cognitive impairment as a diagnostic entity. J Int Med 2004;256:183-194.

Petersen RC, Morris JC. Mild cognitive impairment as a clinical entity and treatment target. Arch Neurol 2005;62:1160-1163. [PubMed: 16009779]

Petersen RC, Smith GE, Waring SC, Ivnik RJ, Tangalos EG, Kokmen E. Mild cognitive impairment: Clinical characterization and outcome. Arch Neurol 1999;56:303-308. [PubMed: 10190820]

Petersen RC, Doody RS, Kurz A, Mohs RC, Morris JC, Rabins PV, Ritchie K, Rossor MN, Thal LJ, Winblad B. Current concepts in mild cognitive impairment. Arch Neurol 2001;58:1985-1992. [PubMed: 11735772]

Petersen RC, Thomas RG, Grundman M, Bennett D, Doody R, Ferris S, Galasko D, Jin S, Kaye J, Levey A, Pfeiffer E, Sano M, et al. Vitamin E and donepezil for the treatment of mild cognitive impairment. N Engl J Med 2005;52:2379-2388. [PubMed: 15829527]

Petrella JR, Prince SE, Wang L, Hellegers C, Doraiswamy PM. Prognostic value of posteromedial cortex deactivation in mild cognitive impairment. PLoS ONE 2007a;2:e1104. [PubMed: 17971867]

Petrella JR, Wang L, Krishnan S, Slavin MJ, Prince SE, Tran TT, Doraiswamy PM. Cortical deactivation in mild cognitive impairment: High-field-strength functional MR imaging. Radiology 2007b; 245:224-235. [PubMed: 17885190]

Pfefferbaum A, Sullivan EV, Hedehus M, Lim KO, Adalsteinsson E, Moseley M. Age-related decline in brain white matter anisotropy measured with spatially corrected echoplanar diffusion tensor imaging. Magn Reson Med 2000;44:259-268. [PubMed: 10918325]

Poettrich K, Weiss PH, Werner A, Lux S, Donix M, Gerber J, von Kummer R, Fink GR, Holthoff VA. Altered neural network supporting declarative long-term memory in mild cognitive impairment. Neurobiol Aging 2009;30:284-298. [PubMed: 17644222]

Portet F, Ousset PJ, Visser PJ, Frisoni GB, Nobili F, Scheltens P, Vellas B, Touchon J. the MCI Working Group of the European Consortium on Alzheimer's Disease (EADC). Mild cognitive impairment (MCI) in medical practice: A critical review of the concept and new diagnostic procedure. Report of 
the MCI Working Group of the European Consortium on Alzheimer's Disease. J Neurol Neurosurg Psychiatry 2006;77:714-718. [PubMed: 16549412]

Rasquin SMC, Lodder J, Visser PJ, Lousberg R, Verhey FRJ. Predictive accuracy of MCI subtypes for Alzheimer's disease and vascular dementia in subjects with mild cognitive impairment: A 2-year follow-up study. Dement Geriatr Cogn Disord 2005;19:113-119. [PubMed: 15591801]

Ravaglia G, Forti P, Maioli F, Martelli M, Servadei L, Brunetti N, Pantieri G, Mariani E. Conversion of mild cognitive impairment to dementia: Predictive role of mild cognitive impairment subtypes and vascular risk factors. Dement Geriatr Cogn Disord 2006;21:51-58. [PubMed: 16276110]

Raz N, Rodrigue KM. Differential aging of the brain: Patterns, cognitive correlates and modifiers. Neurosci Biobehav Rev 2006;30:730-748. [PubMed: 16919333]

Reiman EM, Caselli RJ, Yun LS, Chen K, Bandy D, Minoshima S, Thibodeau SN, Osborne D. Preclinical evidence of Alzheimer's disease in persons homozygous for the epsilon 4 allele for apolipoprotein E. N Engl J Med 1996;334:752-758. [PubMed: 8592548]

Reiman EM, Chen K, Alexander GE, Caselli RJ, Bandy D, Osborne D, Saunders AM, Hardy J. Functional brain abnormalities in young adults at genetic risk for late-onset Alzheimer's dementia. Proc Natl Acad Sci USA 2004;101:284-289. [PubMed: 14688411]

Reiman EM, Chen K, Alexander GE, Caselli RJ, Bandy D, Osborne D, Saunders AM, Hardy J. Correlations between apolipoprotein E epsilon4 gene dose and brain-imaging measurements of regional hypometabolism. Proc Natl Acad Sci USA 2005;102:8299-8302. [PubMed: 15932949]

Ries ML, Jabbar BM, Schmitz TW, Trivedi MA, Gleason CE, Carlsson CM, Rowley HA, Asthana S, Johnson SC. Anosognosia in mild cognitive impairment: Relationship to activation of cortical midline structures involved in self-appraisal. J Int Neuropsychol Soc 2007;13:450-461. [PubMed: 17445294]

Rose SE, Chen F, Chalk JB, Zelaya FO, Strugnell WE, Benson M, Semple J, Doddrell DM. Loss of connectivity in Alzheimer's disease: An evaluation of white matter tract integrity with colour coded MR diffusion tensor imaging. J Neurol Neurosurg Psychiatry 2000;69:528-530. [PubMed: 10990518]

Rose SE, McMahon KL, Janke AL, O’Dowd B, de Zubicaray G, Strudwick MW, Chalk JB. Diffusion indices on magnetic resonance imaging and neuropsychological performance in amnestic mild cognitive impairment. J Neurol Neurosurg Psychaitry 2006;77:1122-1128.

Rountree SD, Waring SC, Chan WC, Lupo PJ, Darby EJ, Doody RS. Importance of subtle amnestic and nonamnestic deficits in mild cognitive impairment: Prognosis and conversion to dementia. Dement Geriatr Cogn Disord 2007;24:476-482. [PubMed: 17992015]

Rozzini L, Chilovi BV, Conti M, Delrio I, Borroni B, Trabucchi M, Padovani A. Neuropsychiatric symptoms in amnestic and nonamnestic mild cognitive impairment. Dement Geriatr Cogn Disord 2008;25:32-36. [PubMed: 18025827]

Seo SW, Im K, Lee J, Kim Y, Kim ST, Kim SY, Yang DW, Kim SI, Cho YS, Na DL. Cortical thickness in single- versus multiple-domain amnestic mild cognitive impairment. NeuroImage 2007;36:289297. [PubMed: 17459730]

Smith, GE.; Ivnik, RJ. Normative neuropsychology. In: Petersen, RC., editor. Mild Cognitive Impairment: Aging to Alzheimer's Disease. Oxford University Press; New York: 2003. p. 63-88.

Sperling R. Functional MRI studies of associative encoding in normal aging, mild cognitive impairment, and Alzheimer's disease. Ann NY Acad Sci 2007;1097:146-155. [PubMed: 17413017]

Sullivan EV, Pfefferbaum A. Diffusion tensor imaging and aging. Neurosci Biobehav Rev 2006;30:749761. [PubMed: 16887187]

Tabert MH, Manly JJ, Liu X, Pelton GH, Rosenblum S, Jacobs M, Zamora D, Goodkind M, Bell K, Stern Y, Devanand DP. Neuropsychological prediction of conversion to alzheimer disease in patients with mild cognitive impairment. Arch Gen Psychiatry 2006;63:916-924. [PubMed: 16894068]

Takahashi S, Yonezawa H, Takahashi J, Kudo M, Inoue T, Tohgi H. Selective reduction of diffusion anisotropy in white matter of Alzheimer disease brains measured by 3.0 Tesla magnetic resonance imaging. Neurosci Lett 2002;332:45-48. [PubMed: 12377381]

Tam C, Lam L, Chiu HFK, Lui VWC. Characteristic Profiles of Instrumental Activities of Daily Living in Chinese Older Persons with Mild Cognitive Impairment. Am J Alzheimer's Dis Other Dement 2007;22:211-217. 
Teng E, Lu PH, Cummings JL. Deficits in facial emotion processing in mild cognitive impairment. Dement Geriatr Cogn Disord 2007;23:271-279. [PubMed: 17351319]

Thal LJ, Ferris SH, Kirby L, Block GA, Lines CR, Yuen E, Assaid C, Nessly ML, Norman BA, Baranak $\mathrm{CC}$, Reines SA. A randomized, double-blind, study of rofecoxib in patients with mild cognitive impairment. Neuropsychopharmacology 2005;30:1204-1215. [PubMed: 15742005]

Ukmar M, Makuc E, Onor ML, Garbin G, Trevisiol M, Cova MA. Evaluation of white matter damage in patients with Alzheimer's disease and in patients with mild cognitive impairment by using diffusion tensor imaging. Radiol Med 2008;113:915-922. [PubMed: 18618077]

Vannini P, Almkvist O, Dierks T, Lehmann C, Wahlund LO. Reduced neuronal efficacy in progressive mild cognitive impairment: A prospective fMRI study on visuospatial processing. Psychiatry Res 2007;156:43-57. [PubMed: 17719211]

Verghese J, Robbins M, Holtzer R, Zimmerman ME, Wang C, Xue X, Lipton RB. Gait dysfunction in mild cognitive impairment syndromes. J Am Geriatr Soc 2008;56:1244-1251. [PubMed: 18482293]

Wadley VG, Crowe M, Marsiske M, Cook SE, Unverzagt FW, Rosenberg AL, et al. Changes in Everyday Function in Individuals with Psychometrically Defined Mild Cognitive Impairment in the Advanced Cognitive Training for Independent and Vital Elderly Study. J Am Geriatr Soc 2007;55:1192-1198. [PubMed: 17661957]

Westervelt HJ, Bruce JM, Coon WG, Tremont G. Odor identification in mild cognitive impairment subtypes. J Clin Exp Neuropsychol 2008;30:151-156. [PubMed: 18938667]

Whitwell JL, Przybelski SA, Weigand SD, Knopman D, Boeve BF, Petersen RC, Jack CR. 3D maps from multiple MRI illustrate changing atrophy patterns as subjects progress from mild cognitive impairment to Alzheimer's disease. Brain 2007;130:1777-1786. [PubMed: 17533169]

Winblad B, Palmer K, Kivipelto M, Jelic V, Fratiglioni L, Wahlund LO, Nordberg A, Backman L, Albert M, Almkvist O, Arai H, Basun H, et al. Mild cognitive impairment-Beyond controversies, towards a consensus: Report of the International Working Group on Mild Cognitive Impairment. J Int Med 2004;256:240-246.

Winblad B, Gauthier S, Scinto L, Feldman H, Wilcock GK, Truyen L, Mayorga AJ, Wang D, Brashear HR, Nye JS. Safety and efficacy of galantamine in subjects with mild cognitive impairment. Neurology 2008;70:2024-2035. [PubMed: 18322263]

Wolf H, Jelic V, Gertz HJ, Nordberg A, Julin P, Wahlund LO. A critical discussion of the role of neuroimaging in mild cognitive impairment. Acta Neurol Scand Suppl 2003;179:52-76. [PubMed: 12603252]

Yaffe K, Petersen RC, Lindquist K, Kramer J, Miller B. Subtype of mild cognitive impairment and progression to dementia and death. Dement Geriatr Cogn Disord 2006;22:312-319. [PubMed: 16940725]

Yetkin FZ, Rosenberg RN, Weiner MF, Purdy PD, Cullum CM. FMRI of working memory in patients with mild cognitive impairment and probable Alzheimer's disease. Eur Radiol 2006;16:193-206. [PubMed: 16402259]

Zanetti M, Ballabio C, Abbate C, Cutaia C, Vergani C, Bergamaschini L. Mild cognitive impairment subtypes and vascular dementia in community-dwelling elderly people: A 3-year follow-up study. J Am Geriatr Soc 2006;54:580-586. [PubMed: 16686866]

Zhang Y, Schuff N, Jahng GH, Bayne W, Mori S, Schad L, Mueller S, Du AT, Kramer JH, Yaffe K, Chui HC, Jagust WJ, et al. Diffusion tensor imaging of cingulum fibers in mild cognitive impairment and Alzheimer disease. Neurology 2007;68:13-19. [PubMed: 17200485] 


\section{TABLE I}

Clinical Trials in aMCI

\begin{tabular}{lllll}
\hline Agent & $\boldsymbol{N}$ & Duration & Endpoint & Outcome \\
\hline Donepezil & 269 & 24 weeks & Symptoms & Negative \\
Donepezil/Vitamin E & 769 & 3 years & AD & Partially positive \\
Rofecoxib & 1200 & $2-3$ years & AD & Negative \\
Galantamine & 995 & 2 years & CDR 1 & Negative \\
& 1062 & 2 years & CDR 1 & Negative \\
Rivastigmine & 1018 & $3-4$ years & AD & Negative \\
\hline
\end{tabular}

$\mathrm{AD}$, Alzheimer's disease; CDR, clinical dementia rating. 